\title{
HORMIGONES CON CENIZA DE CASCARA DE ARROZ (R.H.A.): INFLUENCIA DEL CURADO Y DEL AGUA DE AMASADO
}

\author{
(CONCRETES WITH RICE HUSK ASHES [R.H.A.]: CURING AND MIXING WATER INFLUENCE)
}

Julián Salas, Dr. Ing. Ind., IETcc - CSIC (España)

Gabriel Gómez, Ing. Civil (Colombia)

Janer Veras, Ing. Civil (República Dominicana)

Equipo "Viviendas de Muy Bajo Coste», IETcC - CSIC

$684-11$

\section{RESUMEN}

Se busca establecer la influencia de cinco tipos de curado sobre las resistencias a compresión de probetas cilíndricas de hormigón, de 7,5 × 15,0 cm, en las que se ha variado la proporción de cemento y ceniza de cascarilla de arroz o R.H.A. en el material ligante $y$, por otra parte, se trata de conseguir la relación óptima de agua/conglomerante, tratando de obtener una mayor resistencia a compresión de las probetas e igual manejabilidad.

\section{SUMMARY}

It is about to find out and fix the influence of five types of curing on the compression resistanbces in concrete cylindrical samples of 7,5 × 15,0 cm, where cement and rice husk ashes proportion has been changed in the binding material; on other hand it is about to reach an optimun water/binding material ratio to obtain a stronger resistance to compression of the samples and the same workability.

\section{ANTECEDENTES}

Como parte del programa del proyecto de investigación: "MATERIALES, TECNOLOGIAS Y PROTOTIPOS DE VIVIENDAS DE MUY BAJO COSTE", que se desarrolla en el I.E.T.c.c., en este trabajo se plantea el estudio de la influencia de cinco tipos de curado, sobre la resistencia a compresión de probetas cilíndricas de hormigón, elaboradas con tres proporciones diferentes de material ligante (cemento-ceniza): $100 \%-0 ; 67 \%-33 \% ; 50 \%-50 \%$, e igual relación agua/conglomerante. Además se estudia el comportamiento de estos hormigones, al variar la cantidad de agua de amasado para igual manejabilidad.

Existe abundante bibliografia y antecedentes sobre la influencia que ejerce el tipo de curado sobre las resistencias mecánicas, la durabilidad y el comportamiento general de los hormigones normales (1).
De hecho, hay patrones aceptados de variación de valores en resistencias para hormigones normales, los cuales, en determinadas condiciones, mejoran con el aumento del contenido de humedad, temperatura y presión del ambiente donde se efectúa el curado. No obstante, los autores desconocen, a pesar del volumen importante de bibliografía consultada, artículos en el sentido de establecer los efectos de curado, sobre hormigones elaborados con R.H.A., efectos que se presuponen importantes, ya que se trata de un producto con características puzolánicas, con su típica lentitud en la consecución de resistencias a edades tempranas.

Con anterioridad (2), se habia analizado la variación de la resistencia a compresión de cubos de mortero de $7 \mathrm{~cm}$, a base de diferentes proporciones cemento/ceniza y, siguiendo directrices similares, se adoptaron las proporciones arriba mencionadas de $100 \%-0$; $67 \%-33 \% ; 50 \%-50 \%$. 
La dosificación del hormigón testigo, o dosificación patrón de origen, se estableció guardando coherencia con la empleada en otros trabajos del mismo proyecto de investigación (3).

Posteriormente, a la vista de los bajos valores de manejabilidad obtenidos (medida mediante asentamiento de cono de Abrams), se realizaron otras pruebas con relación agua/conglomerante variable, al objeto de obtener la misma manejabilidad, circunstancia que se presenta más acorde con la realidad del trabajo en la práctica.

Se optó por realizar probetas cilindricas de 7,5 $\times 15 \mathrm{~cm}$, por dos razones. En primer lugar, por economia de materiales y facilidad de manejo, y en segundo lugar, porque al tratarse de resultados comparativos, se estimó que afectarian a todas las amasadas en forma aproximadamente semejante (4).

Adicionalmente, la granulometria de los áridos (D. máx.: $2,5 \mathrm{~cm}$ ) permite cumplir la recomendación de que el diámetro de la probeta sea, por lo menos, tres veces el tamaño máximo del árido y la tendencia actual, en tecnologia de ensayos de hormigón, deriva hacia ese tamaño de probetas, mostrando que los resultados obtenidos ofrecen una dispersión similar y unos valores de resistencia ligeramente menores, contrario a las ideas que, hasta hace algunos años, predominaban al respecto (4).

\section{CARACTERISTICAS DE LOS MATERIALES EMPLEADOS}

\subsection{Cemento}

Se empleó un cemento Portland de alta resistencia inicial ARII-450, con las siguientes caracteristicas:

\begin{tabular}{l|cl}
\hline \multicolumn{1}{c|}{ Cemento } & \multicolumn{2}{|c}{ ARl-450 } \\
\hline $\begin{array}{l}\text { Finura (Permeabilimetro de } \\
\text { Blaine) }\end{array}$ & 4392 & $\mathrm{~cm}^{2} / \mathrm{gr} \mathrm{B}$ \\
$\begin{array}{l}\text { Peso especifico } \\
\text { Fraguado inicial (Aguja de } \\
\text { Vicat) }\end{array}$ & $2 \mathrm{~h} .22$ & $\mathrm{gr} / \mathrm{cm}^{3}$ \\
$\begin{array}{l}\text { Fraguado final (Aguja de } \\
\text { Vicat) }\end{array}$ & $3 \mathrm{~h} .27$ \\
$\begin{array}{l}\text { Expansión en autoclave } \\
\text { Ex }\end{array}$ & $-1 \mathrm{~mm}$ \\
\hline
\end{tabular}

\subsection{Ceniza}

Obtenida según el procedimiento descrito en la referencia (2), mostraba las siguientes caracteristicas:

\section{Composición química:}

Pérdida al fuego ........................

Residuo soluble ................... 0,17

Anhidrido Silicico, $\mathrm{SiO}_{2} \ldots \ldots \ldots \ldots \ldots . \quad 91,26$

Oxido Aluminico, $\mathrm{Al}_{2} \mathrm{O}_{3} \ldots \ldots \ldots \ldots \ldots \ldots . . \ldots, 0,94$

Oxido Férrico, $\mathrm{Fe}_{2} \mathrm{O}_{3} \ldots \ldots \ldots \ldots \ldots \ldots \ldots, 0,37$

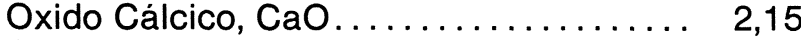

Oxido, Magnésico, MgO............... 0,88

\section{Caracteristicas físicas:}

Finura (Permeabili-

metro de Blaine) ... $3518 \mathrm{~cm}^{2} / \mathrm{gr}$ a $4200 \mathrm{~cm}^{2} / \mathrm{gr}$ Peso especifico.... 2, $2,05 \mathrm{gr} / \mathrm{cm}^{3}$ Densidad aparente. $\quad 0,7 \mathrm{gr} / \mathrm{cm}^{3}$

\begin{tabular}{|c|c|c|c|c|}
\hline FRAGUADO: & INICIAL & RETRASO & FINAL & RETRASO \\
\hline , CONTROL & $2^{\mathrm{h}} 22^{\prime}$ & 0 & $3^{h}$ & 0 \\
\hline Con $33 \%$ RHA & $4^{\mathrm{h}} 01^{\prime}$ & $1^{\mathrm{h}} 39^{\prime}$ & $5^{\mathrm{h}} 05^{\prime}$ & $2^{h} 05^{\prime}$ \\
\hline Con $50 \%$ RHA & $4^{\mathrm{h}} 42^{\prime}$ & $2^{\mathrm{h}} 20^{\prime}$ & $6^{\mathrm{h}} 17 \mathrm{r}$ & $3^{\mathrm{h}} 17 \mathrm{r}$ \\
\hline
\end{tabular}

AUTOCLAVE:

\begin{tabular}{|c|c|c|c|}
\cline { 2 - 4 } \multicolumn{1}{c|}{} & ANTES & DESPUES & EXPANSION \\
\hline Con 33 \% RHA & 2,58 & 3,17 & 0,23 \\
\hline Con 50\% RHA & 1,39 & 1,44 & 0,01 \\
\hline
\end{tabular}

\begin{tabular}{|l|c|c|c|c|}
\cline { 2 - 5 } AGUJAS: & $\begin{array}{c}\text { MEDIDA A } \\
24^{\mathrm{h}}\end{array}$ & $\begin{array}{c}\text { MEDIDA A } \\
3^{\mathrm{h}}\end{array}$ & $\begin{array}{c}\text { MEDIDA A } \\
7 \text { DIAS }\end{array}$ & $\begin{array}{c}\text { EXPANSION/ } \\
\text { RETRACCION }\end{array}$ \\
\hline Con 33\% RHA & 25 & 24 & 26 & $-1,5$ RETRACCION \\
\hline Con 50\% RHA & 14 & 12 & 11 & $+0,16$ EXPANSION \\
\hline
\end{tabular}

\subsection{Aridos}

Se utilizaron como áridos una grava procedente del río Jarama y una arena de Segovia, que presentaban las siguientes características físicas en las tablas que aparecen a continuación.

El módulo de finura de la grava es de 7,55 y de 3,13 el de la arena.

Es de resaltar la textura muy pulida del árido grueso, exceso de fino y la presencia de apreciable proporción de partículas planas y alargadas, aspectos que conducen a que su comportamiento de adherencia con el mortero no sea óptima, con la consecuente disminución de las resistencias mecánicas (Ver Fig. 1). 


\begin{tabular}{|c|r|r|r|r|r|r|}
\hline \multirow{2}{*}{ ABERTURA } & \multicolumn{4}{|c|}{ CANTIDAD RETENIDA POR TAMICES } & \multicolumn{2}{|c|}{ \% RETENIDO } \\
\cline { 2 - 7 } & \multicolumn{1}{|c|}{1} & \multicolumn{1}{|c|}{2} & \multicolumn{1}{c|}{3} & \multicolumn{1}{c|}{ MEDIA } & Parcial & Acumulado \\
\hline 19,05 & 0,00 & 0,00 & 0,00 & 0,00 & 0,00 & 0,00 \\
12,00 & 927,89 & 970,00 & 942,20 & 949,00 & 18,98 & 18,98 \\
9,11 & 1205,08 & 1216,00 & 1236,00 & 1219,27 & 24,39 & 43,37 \\
4,76 & 2498,10 & 2458,00 & 2458,30 & 2469,13 & 49,39 & 92,76 \\
2,00 & 348,10 & 343,88 & 323,75 & 338,58 & 6,77 & 99,53 \\
Fondo & 20,00 & 19,10 & 31,70 & 23,60 & 0,47 & 100,00 \\
$\Sigma$ & 4999,89 & 4999,98 & 4998,95 & 4999,61 & & \\
\hline
\end{tabular}

Granulometría de la Grava de una muestra de $5 \mathrm{~kg}$ tomada mediante procedimientos cuantiles.

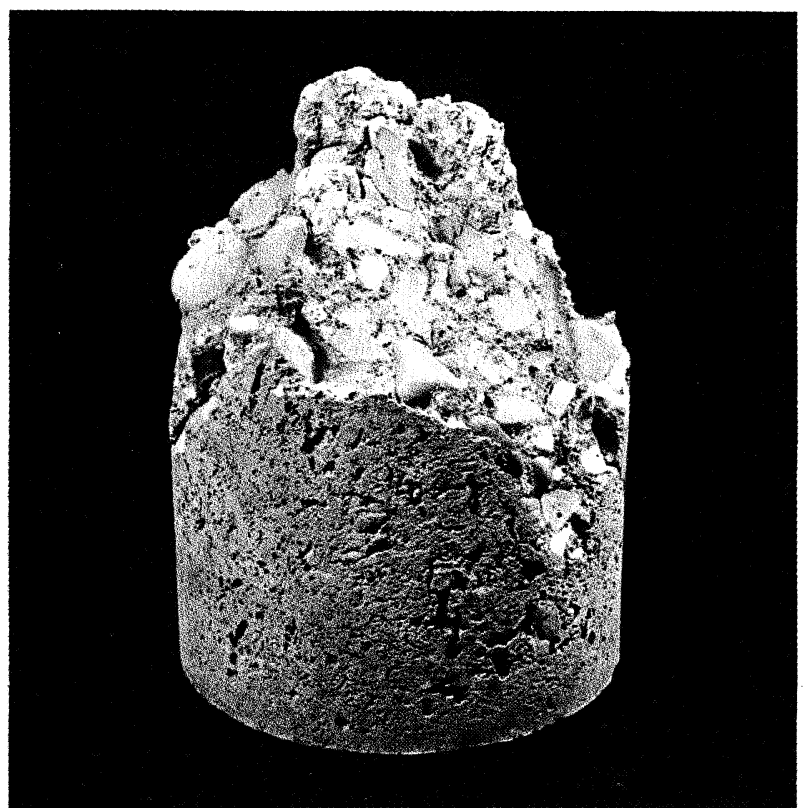

Fig. 1

\section{METODOLOGIA DE LOS ENSAYOS}

En una báscula Roch, de capacidad máxima $230 \mathrm{kgs}$ y aproximación $0,05 \mathrm{kgs}$, se efectuaba la pesada de los diferentes materiales. Se mezclaban, en primer lugar, los áridos y el cemento en una hormigonera UNACON, con una valocidad de 26 RPM, durante aproximadamente 2 minutos, posteriormente se añadia agua, mezclando 2 minutos adicionales. Se detenía la hormigonera, se limpiaba el material adherido a las paredes y se volvia a mezclar durante, aproximadamente, 1 minuto; luego se efectuaba el ensayo de asentamiento, en cono de Abrams y en los casos de relación $A / C$ variable, se procedia a agregar agua y mezclar durante, aproximadamente 40 segundos, hasta obtener el asentamiento deseado.

Posteriormente se procedia a compactar manualmente, mediante barra, 15 probetas de las dimensiones anteriormente mencionadas $(7,5 \times 15 \mathrm{~cm})$, en tres capas compactadas cada una con 25 golpes (según norma). Con el fin de lograr una adecuada compactación de la última capa, especialmente en las mezclas con alto contenido de ceniza, que presentan una baja

\begin{tabular}{|l|r|r|r|r|r|r|}
\hline \multirow{2}{*}{$\begin{array}{c}\text { ABERTURA } \\
\text { TAMIZ MM }\end{array}$} & \multicolumn{4}{|c|}{ CANTIDAD RETENIDA POR TAMICES } & \multicolumn{2}{c|}{ \% RETENIDO } \\
\cline { 2 - 7 } & \multicolumn{1}{|c|}{1} & \multicolumn{1}{|c|}{3} & \multicolumn{1}{c|}{ MEDIA } & Parcial & Acumulado \\
\hline 9,11 & 0,00 & 0,00 & 0,00 & 0,00 & 0,00 & 0,00 \\
4,76 & 38,80 & 40,10 & 41,09 & 40,00 & 1,60 & 1,60 \\
2,36 & 377,40 & 409,00 & 413,50 & 399,97 & 16,02 & 17,62 \\
1,19 & 436,20 & 468,00 & 473,80 & 459,33 & 18,40 & 36,02 \\
0,59 & 837,90 & 848,30 & 873,70 & 839,97 & 33,64 & 69,66 \\
0,29 & 548,90 & 510,00 & 509,80 & 522,90 & 20,94 & 90,60 \\
0,149 & 182,80 & 157,00 & 156,00 & 165,27 & 6,62 & 97,22 \\
Fondo & 70,80 & 67,10 & 70,00 & 69,30 & 2,78 & 100,00 \\
$\Sigma$ & 2490,8 & 2499,5 & 2497,9 & 2497 & & \\
\hline
\end{tabular}

Granulometría de la arena de una muestra de 2,5 kgs.

manejabilidad por la alta absorción de agua en este material, se colocaba un "collarin" suplementario. Posteriormente se enrasaba la última capa y se procedia al refrentado con cemento.

Al cumplir 24 horas, eran desmoldadas las probetas, marcadas y pesadas individualmente y almacenadas en el ambiente del curado escogido. A las edades establecidas $(3 ; 7 ; 28$; 60 y 90 dias) eran retiradas del respectivo ambiente, pesadas y ensayadas a compresión en una máquina Universal AMSLER, con una escala de capacidad máxima de 25 toneladas y aproximación de $50 \mathrm{kgs}$, modificando el tiempo de ensayo para garantizar que se estaba dentro de los límites de velocidad de aplicación de carga $6 \div 4 \mathrm{kp} / \mathrm{cm}^{2}$, registrándose la carga máxima obtenida.

\section{DESCRIPCION DE LOS TIPOS DE CURADO EMPLEADOS (Fig. 2)}

Como ya se ha apuntado, son cinco los tipos de curado a los que se han sometido idénticas series de probetas:

a) Curado normalizado: cámara de curado a temperatura y humedad constante: $210 \mathrm{C} \pm$ $\pm 10 \mathrm{C}$ y $95 \% \pm 3 \%$ H.R.

b) Curado ambiente interior: condiciones relativamente estables del laboratorio de hormigones del IETCC: $180 \mathrm{C} \pm 20 \mathrm{C} \mathrm{y}$ humedad relativa del $60 \% \pm 5 \%$.

Diariamente, durante la primera semana, las probetas se sometieron a un rociado de agua, mediante vaporizador manual, del orden de un litro de agua/dia, por lote de quince probetas.

c) Curado acelerado: trata de reproducir las condiciones "típicas" del curado acelerado, utilizado en prefabricación de elementos. Mediante depósitos aislados de agua calentada y regulada por termostato, se han seguido los procesos temperatura-tiempo, que se recogen en la Fig. 3 . 


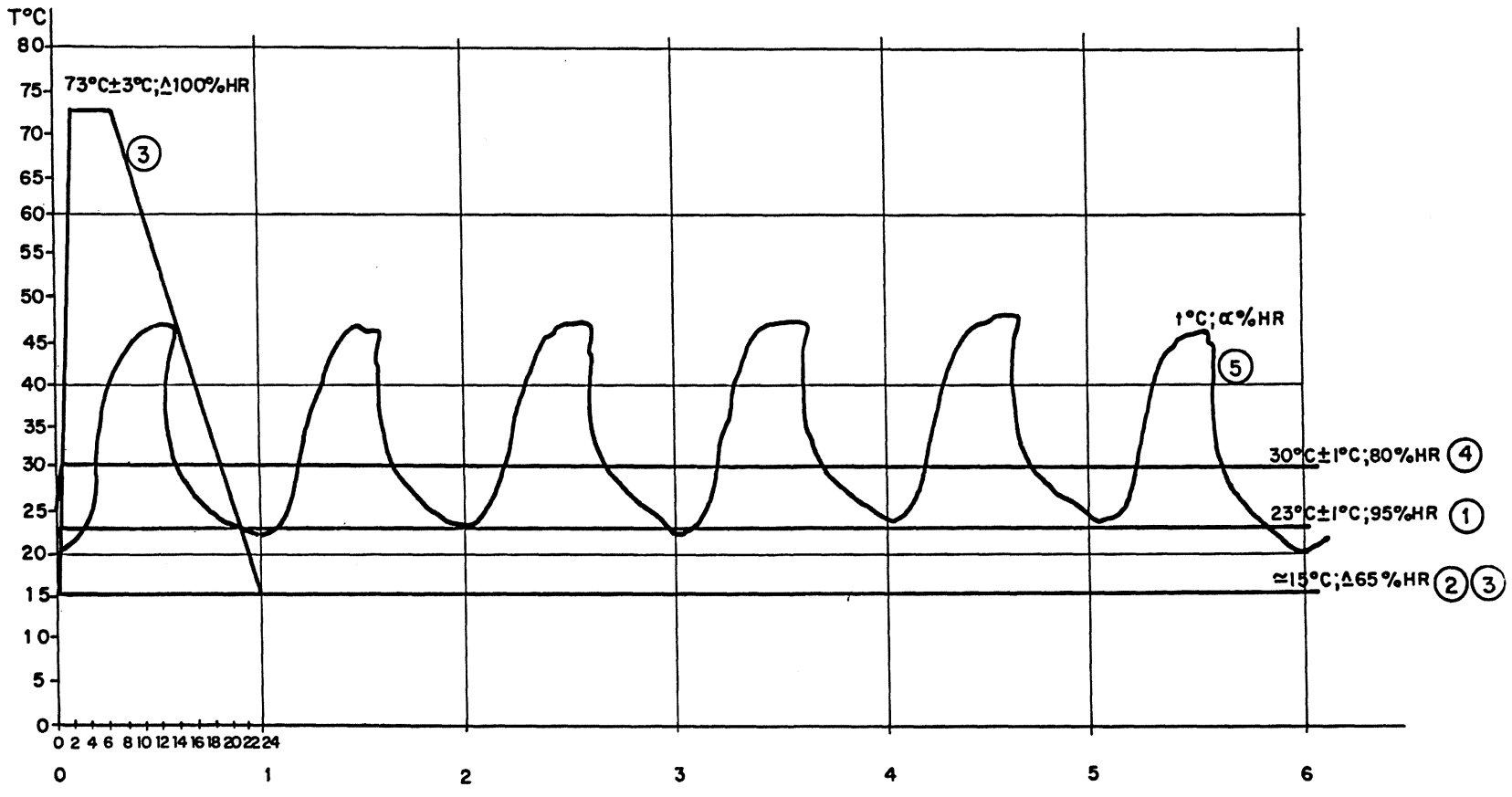

Fig. 2. - Gráficas de la evolución temperaturas-tiempos de los cinco tipos de curado estudiados.

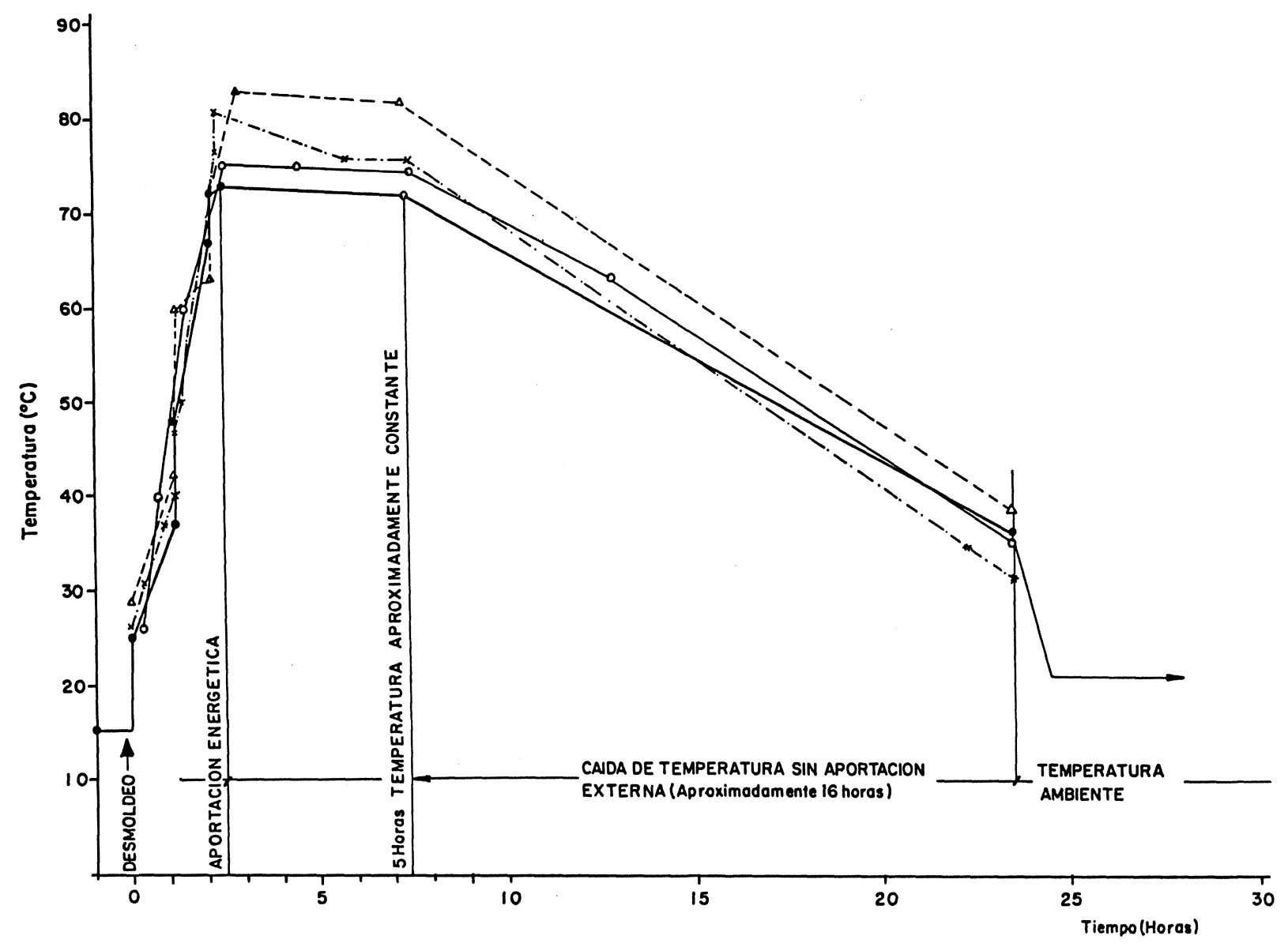

Fig. 3. - Gráficas temperaturas-tiempos del curado acelerado. 
Partiendo de la temperatura ambiente en la zona de hormigonado, se desmoldaron las probetas a las 24 horas, subiendo paulatinamente en dos horas hasta $75 \circ \mathrm{C} \pm$ 5.0 C; se mantuvo prácticamente estable esta temperatura durante cinco horas, momento en el cual se interrumpió la aportación energética externa, por lo que descendia lentamente la temperatura interna, llegando en un periodo de unas 17 horas hasta $\simeq 30 \pm 5.0 \mathrm{C}$, momento en que se sacaron las probetas, pasando a las condiciones descritas como "curado en ambiente interior". Todo el proceso de curado descrito se realiza en un ambiente saturado de humedad.

d) Curado "Caribe»: mediante cámara de curado regulable se han fijado condiciones constantes de $30 \circ \mathrm{C} \pm 1.0 \mathrm{C}$ y $80 \% \pm 3 \%$ de humedad relativa, al objeto de simular un curado a la intemperie, típico de climas tropicales (Ver Fig. 4).

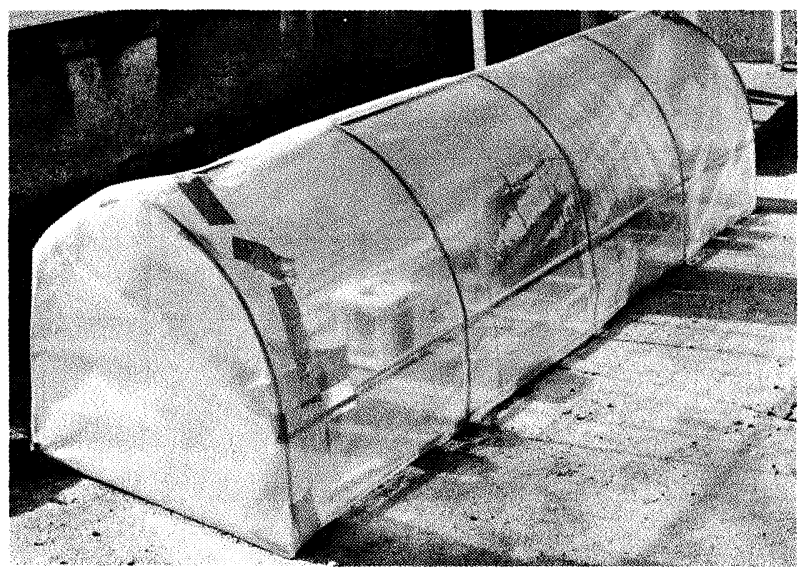

Fig. 5a. - Curado ambiente exterior protegido, o tipo “invernadero", posición cerrado.

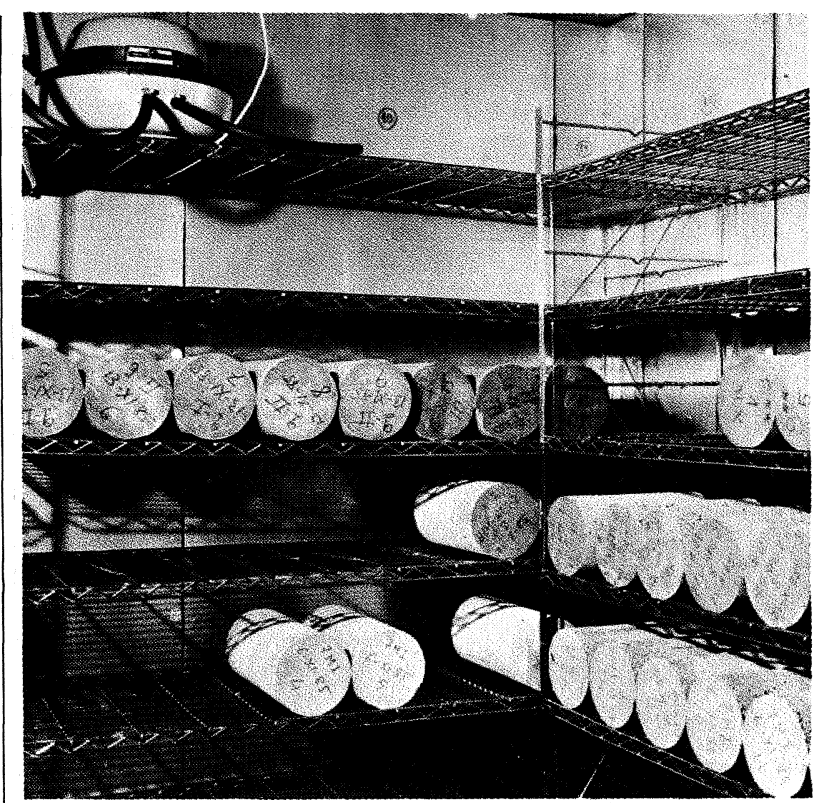

Fig. 4. - Aspecto del interior de la cámara de curado. (Curado normalizado y "caribe").

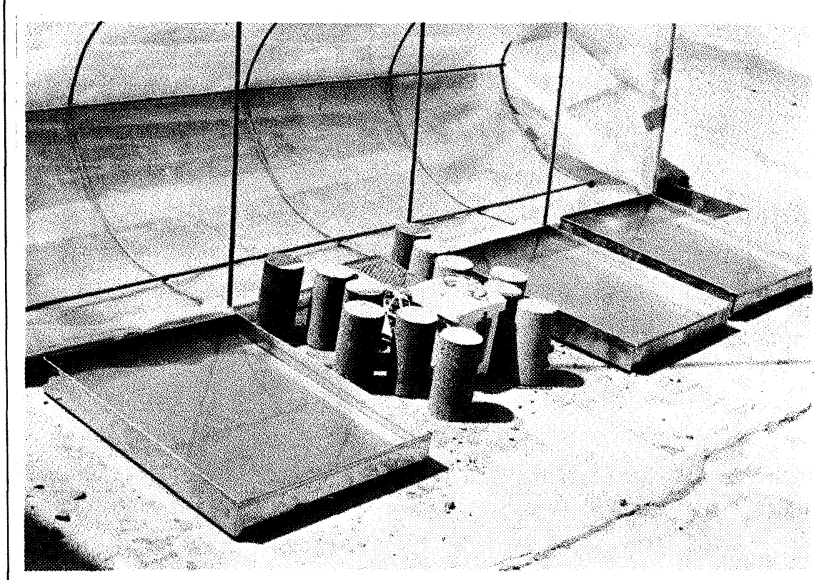

Fig. 5b. - Detalle del interior del "invernadero", superficie de agua, probetas y registrador de H.R. y temperaturas.

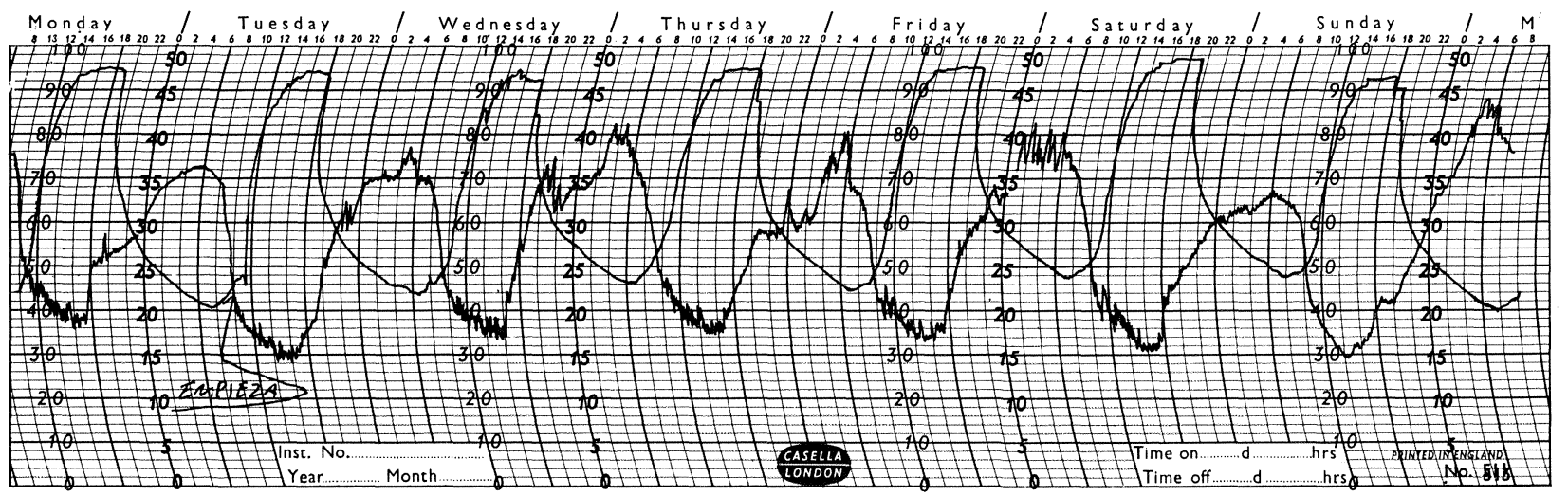

Fig. 6. - Registro semanal de H.R. y temperaturas en el interior del "invernadero". 
e) Curado ambiente exterior protegido: mediante protección a base de plástico transparente y en presencia de abundante agua para su evaporación, se ha realizado el curado en la forma que se indica en las Figs. $5 \mathrm{a}$ y $5 \mathrm{~b}$. En el interior de la zona cubierta, se registró semanalmente la evolución de temperaturas y humedades relativas. Los curados se realizaron durante los meses de junio/julio de 1986, en Madrid, adjuntándose en la Fig. 6, a título de ejemplo, la evolución durante una semana de las temperaturas y humedades relativas en el interior de la zona cubierta. Puede comprobarse que las temperaturas máximas se mantuvieron diariamente entre $450 \div 500 \mathrm{C}$ durante $4 \div 6$ horas y minimos de $200 \mathrm{C} \div 250 \mathrm{C}$. Coinciden los periodos de temperaturas máximas con humedades relativas minimas, del orden del $30 \% \div 40 \%$ de H.R. y las temperaturas mínimas, con humedades máximas del orden de $70 \% \div 10 \%$ de H.R., excepto en dos casos comprendidos entre $60 \% \div$ $70 \%$ de H.R. y $80 \% \div 90 \%$ de H.R. respectivamente.

\section{RESULTADOS PARA CADA TIPO DE CURADO}

\subsection{Introducción}

En todos los casos, se trata de los valores medios de resistencias a compresión de series de tres probetas, expresados en MPa y edades de rotura: $3 ; 7 ; 28 ; 60$ y 90 dias.

La dosificación patrón a la que se aludirá seguidamente en varias ocasiones, responde a las características de un hormigón de baja cuantía en cemento y presenta la siguiente dosificación: $275 \mathrm{~kg}$ de cemento P-ARI-450, $1113 \mathrm{~kg}$ de grava, $756 \mathrm{~kg}$ de arena y 205 litros de agua.

\subsection{Curado normalizado}

Los resultados recogidos en la Fig. 7, muestran cómo los tres conglomerantes, para idéntica relación agua/ $($ cemento + ceniza) $=0,71$, presentan en el tiempo de curado una evolución semejante, aunque con distintas resistencias. Se detectan resultados comparativamente mejores para la proporción 33\% RHA con $67 \%$ P-ARI-450 que para la dosificación con un $50 \%$ de RHA, se muestra incapaz de superar los $10 \mathrm{MPa}$.

La sustitución de un tercio del peso de cemento por RHA en el hormigón adoptado como patrón, puede ser interesante, ya que la caida de resistencias es inferior al $20 \%$ respecto a la del patrón.

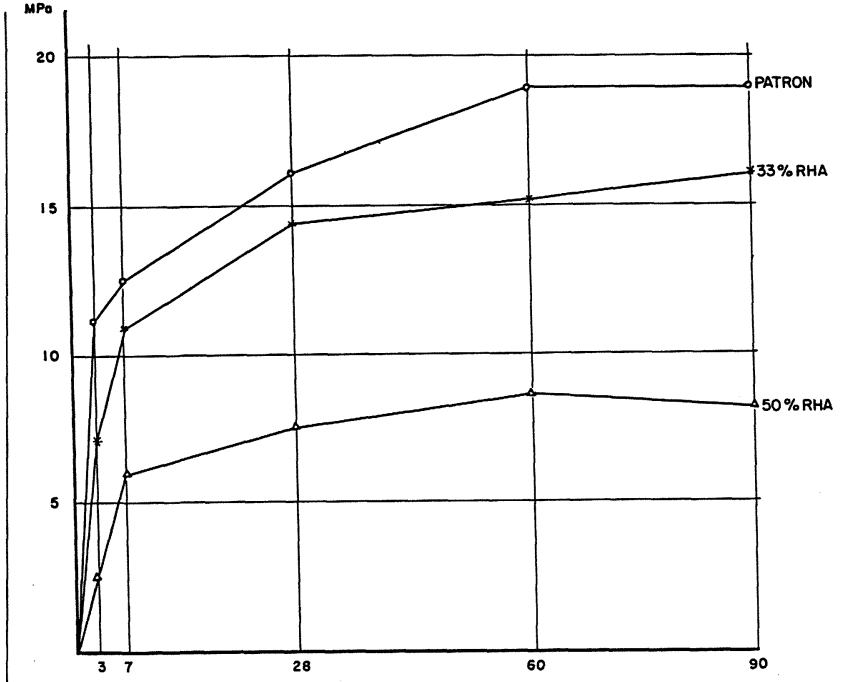

Fig. 7. - Curado normalizado. Relación $a / c=0,71$.

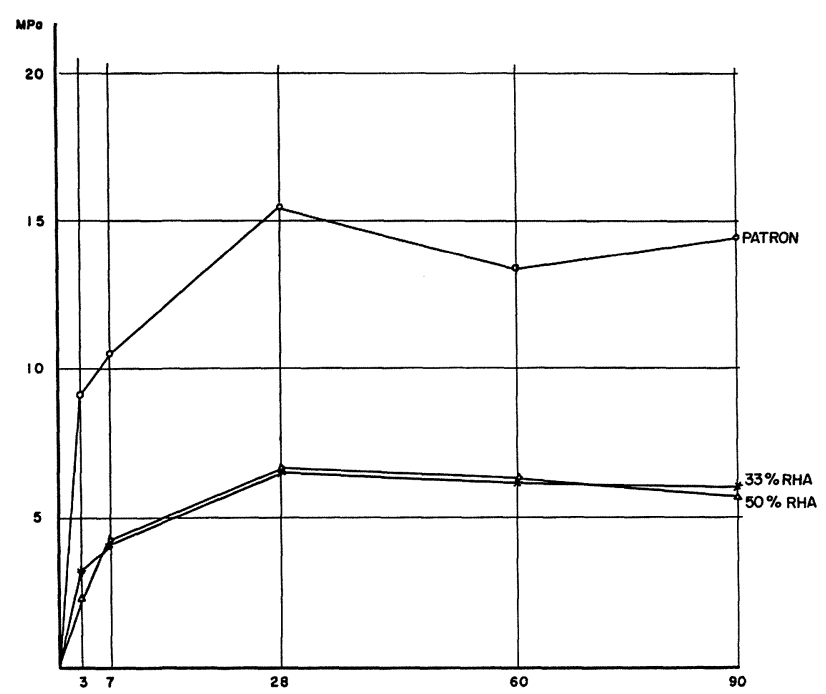

Fig. 8. - Curado ambiente interior $(a / c=0,71)$.

\subsection{Curado ambiente interior}

Los resultados recogidos en la Fig. 8 , manteniendo la relación $\mathrm{a} /(\mathrm{C}+\mathrm{c})=0,71$, son claramente contradictorios, entre otras razones, por no presentar prácticamente diferencias en las resistencias con dosificaciones a base del $50 \%$ ó $33 \%$ de RHA; por la caída de resistencia en la dosificación patrón a 60 dias, asi como por las bajas resistencias a edades tempranas, 3 y 7 dias. Las contradicciones puestas de manifiesto por los resultados a compresión, resultan menos explicables, si se tiene en cuenta, que la temperatura y humedad del local de curado, son prácticamente constantes. 


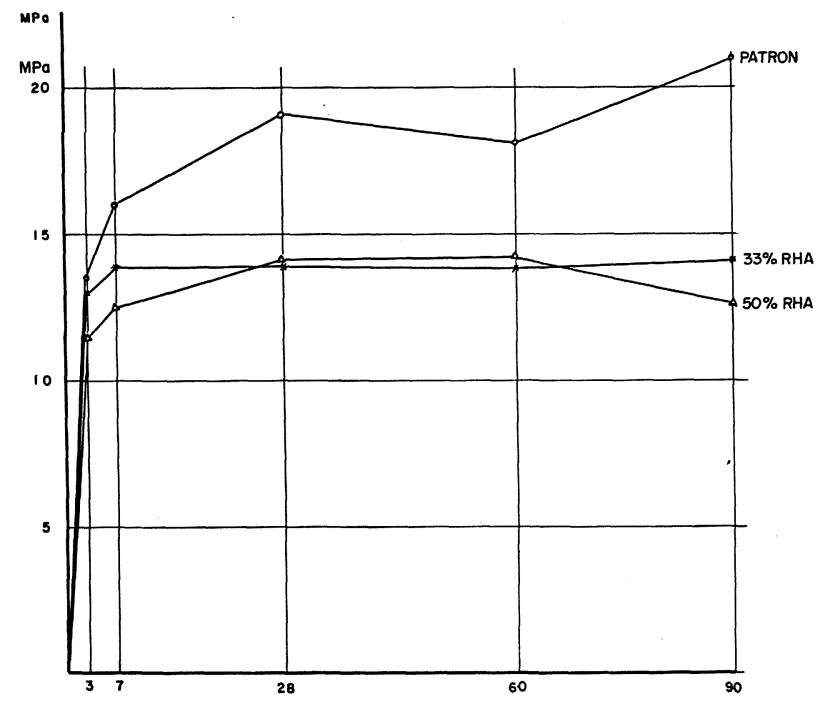

Fig. 9. - Curado acelerado.

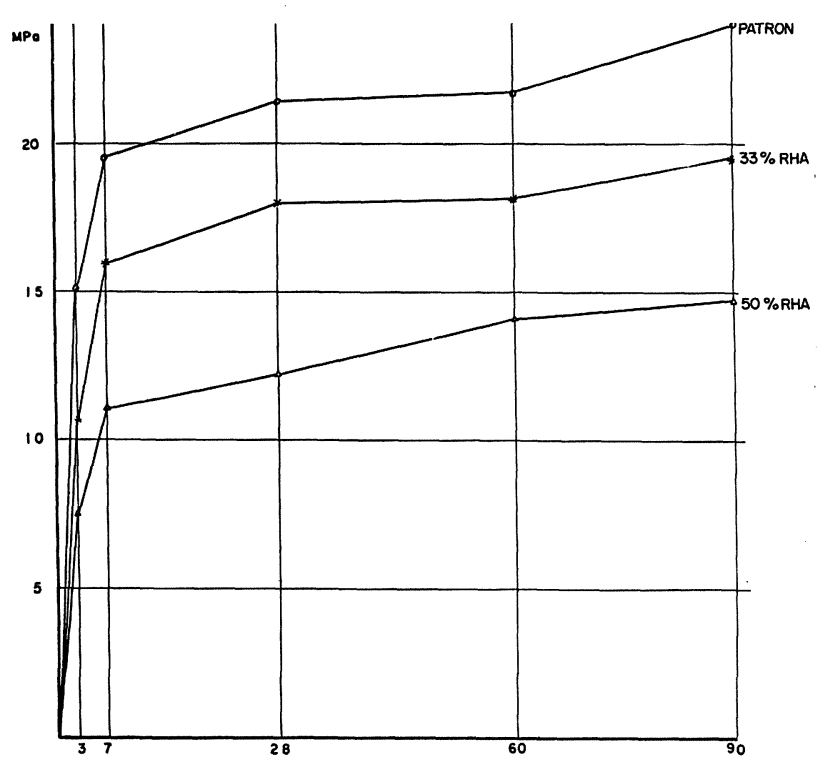

Fig. 10. - Curado "caribe".

\subsection{Curado acelerado (Fig. 9)}

Como era previsible, llaman la atención las altas resistencias conseguidas a edades tempranas -a 3 dias prácticamente el $80 \%$ de la resistencia obtenida a 28 dias, tanto para $33 \%$, como para $50 \%$ de RHA- asi como, el que pasados 28 dias, prácticamente no se experimenten aumentos de resistencias.

Lo que no resultaba previsible es la similitud entre los comportamientos resistentes para $33 \%$ y $50 \%$ de $\mathrm{RHA}$, con resistencias a 28 dias del orden de $14 \mathrm{MPa}$, lo cual puede ser debido a que la ceniza no alcanza a desarrollar su acción puzolánica al reaccionar al inicio la cal del cemento.

\subsection{Curado «caribe»}

Los resultados que se recogen en la Fig. 10, presentan una evolución del aumento de resistencia en el tiempo muy homogéneo (similar a la del curado normalizado, Fig. 6) pero con valores cuantitativos bastante más altos, en el caso del curado "caribe».

El conseguir $7,5 \mathrm{MPa}$ a 3 dias y prácticamente, $15 \mathrm{MPa}$ a 90 dias, a base de un conglomerante con un $50 \%$ de RHA, hacen especialmente viable este conglomerante para determinados usos, en este tipo de climatología de alta temperatura y humedad relativa en forma constante.

La excelente evolución de las resistencias del llamado hormigón "patrón» con este tipo de curado, se materializa en la consecución de resistencias muy superiores (con incrementos del $36 \% ; 56 \%, 34 \%, 15 \%, 26 \%$ ) a todas las edades, respecto del curado normalizado.

\subsection{Curado ambiente exterior protegido}

Los resultados recogidos en la Fig. 11 manifiestan, de forma global, las nefastas consecuencias, en el aspecto de la resistencia a compresión, de este tipo de curado, cuya principal caracteristica es la alternancia «altas temperaturas-baja humedad relativa" con "temperaturas moderadas-alta humedad relativa", según ciclos del tipo de los recogidos en la Fig. 6.

Resultan llamativas las altas, y prácticamente iguales, resistencias conseguidas a 3 dias por los tres tipos de dosificaciones.

El aspecto perjudicial de este tipo de curado, se manifiesta en el hecho de que se mantengan prácticamente inalterables las resistencias a compresión para cualquier edad y dosificación, en el entorno de (10 \pm 2$) \mathrm{MPa}$.

\subsection{Conclusiones parciales}

De lo dicho en este apartado, respecto a los cinco tipos de curado experimentados para las tres dosificaciones, resultan obvias algunas conclusiones:

a) La bondad de los tipos de curado en ambientes con altas cotas de humedad relativa, respecto a los restantes: curados "caribe" y "normalizado". Esto es obvio en los curados "ambiente interior" y "ambiente exterior protegido" e, incluso, en el caso del curado "acelerado", ya que transcurrido el segundo dia se reproducen las condiciones propias del "ambiente interior". 
b) El curado "caribe" se muestra muy superior, incluso al curado "normalizado", para las tres dosificaciones. Ello se logra disminuyendo ligeramente la humedad relativa (del $95 \%$ al $80 \%$ ) y aumentando la temperatura de $(23 \circ \mathrm{C}$ a $30 \circ \mathrm{C})$ respecto del curado «normalizado".

\section{INFLUENCIA DE LOS TIPOS DE CURADO EN LAS RESISTENCIAS A COMPRESION DE LOS HORMIGONES ESTUDIADOS}

\subsection{Introducción}

La presentación de las resistencias a compresión ya comentadas (Figs. 7 a 11) agrupadas ahora según los distintos tipos de dosificación, permiten adelantar algunas conclusiones parciales.

\subsection{Hormigones patrón}

La Fig. 12 muestra de forma clara, la extraordinaria influencia del curado en las resistencias que pueden alcanzarse a compresión, para la misma dosificación.

Con idéntica dosificación, relación agua/cemento y tipo de compactación, actuando únicamente sobre la forma de curado, resultan valores dentro de una zona extraordinariamente amplia. A tres dias, los valores oscilan desde 9,0 MPa (curado "ambiente interior») hasta 15,5 MPa (curado "caribe"). A 90 dias las diferencias siguen siendo manifiestas: $11 \mathrm{MPa}$ para el curado "ambiente exterior protegido" y $24 \mathrm{MPa}$ para el "caribe".

Resulta extraordinariamente adecuado el rendimiento del curado "caribe", que lo hace prometedor para climas con temperaturas y humedad relativas altas y constantes $(\simeq 30 \circ \mathrm{C}$ $y \simeq 80 \%$ H.R.). Se evidencia igualmente lo improcedente que resulta, en este tipo de condiciones climatológicas, el emplear procesos de curado acelerado, lo cual ocurre con frecuencia en la práctica, especialmente en la producción de elementos prefabricados.

\subsection{Hormigones con un $33 \%$ de RHA}

La Fig. 13 muestra la necesidad de curar en ambientes con altas humedades relativas, detectándose resistencias muy bajas para 280 más dias, cuando se someten las probetas al curado de "ambiente interior" y estabilización de la resistencia después de los 28 dias para los casos de curado "ambiente exterior protegido" y "acelerado".

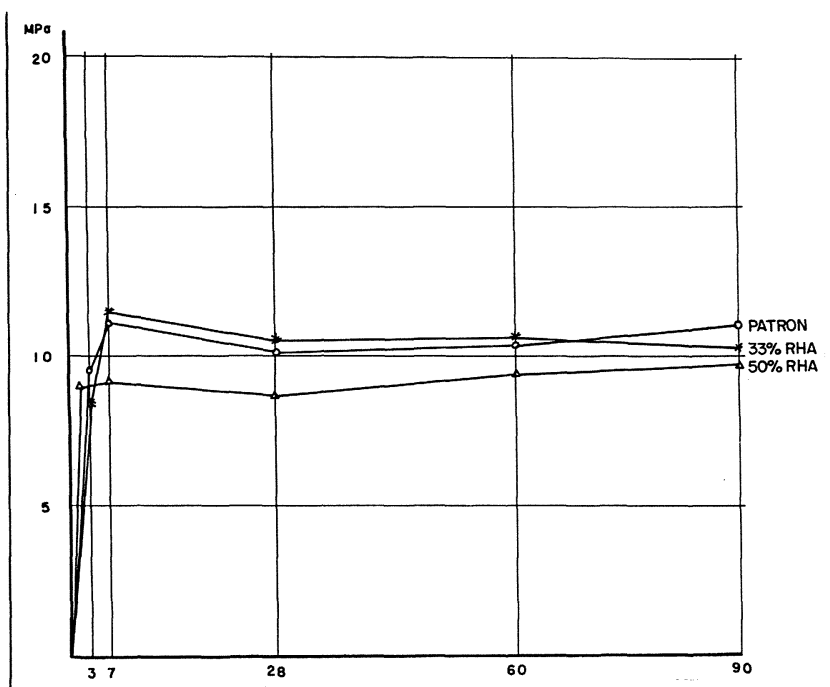

Fig. 11. - Curado ambiente exterior protegido.

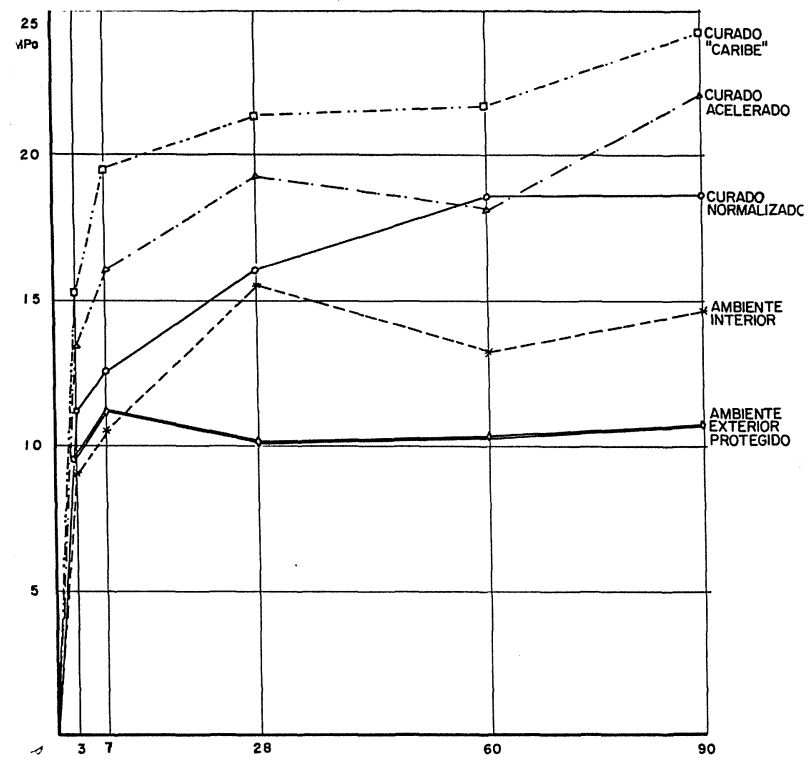

Fig. 12. - Influencia del tipo de curado en el hormigón patrón $(a / c=0,71)$.

Se confirma la consecución de resistencias altas a edades tempranas para el curado "acelerado", asi como el hecho de su, prácticamente estancamiento, después de siete dias. El denominado curado "caribe" sigue manteniendo su primacia para esta dosificación, con $33 \%$ de RHA. Es decir, transcurridos siete dias, se consiguen incrementar en las resistencias a compresión, únicamente en los tipos de curado con alta humedad relativa ("caribe»: $80 \%$ y «normalizado»: $90 \%$ ) manteniéndose prácticamente la resistencia alcanzada a siete dias en el resto: "acelerado" ( $65 \%$ H.R.); "ambiente exterior protegido" (H.R. según ciclos) y "ambiente interior» ( $\simeq 65 \%$ H.R.). 


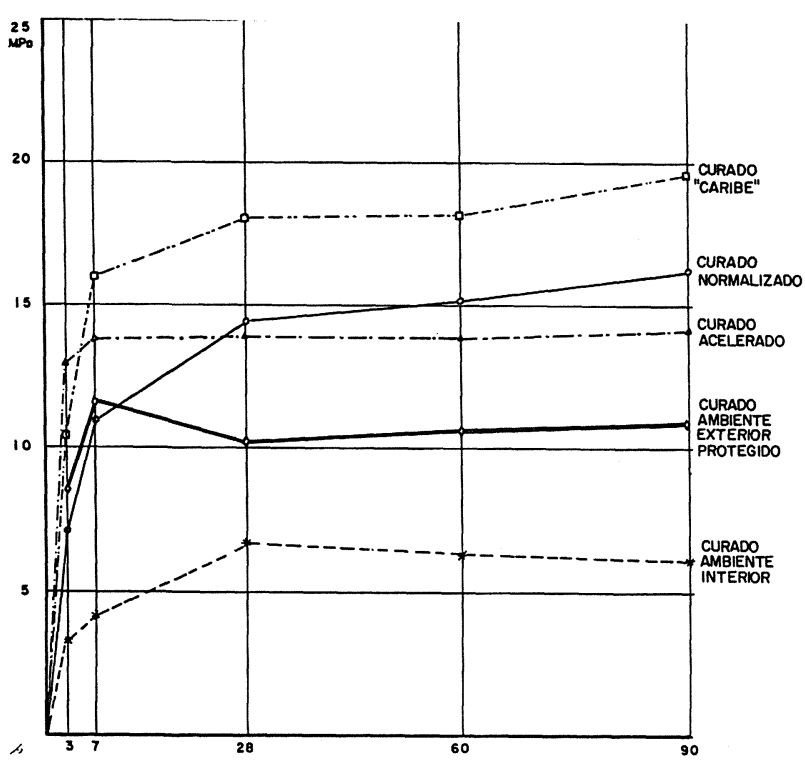

Fig. 13. - Influencia del tipo de curado en hormigón con un $33 \%$ de R.H.A. $(a /[c+c]=0,71)$.

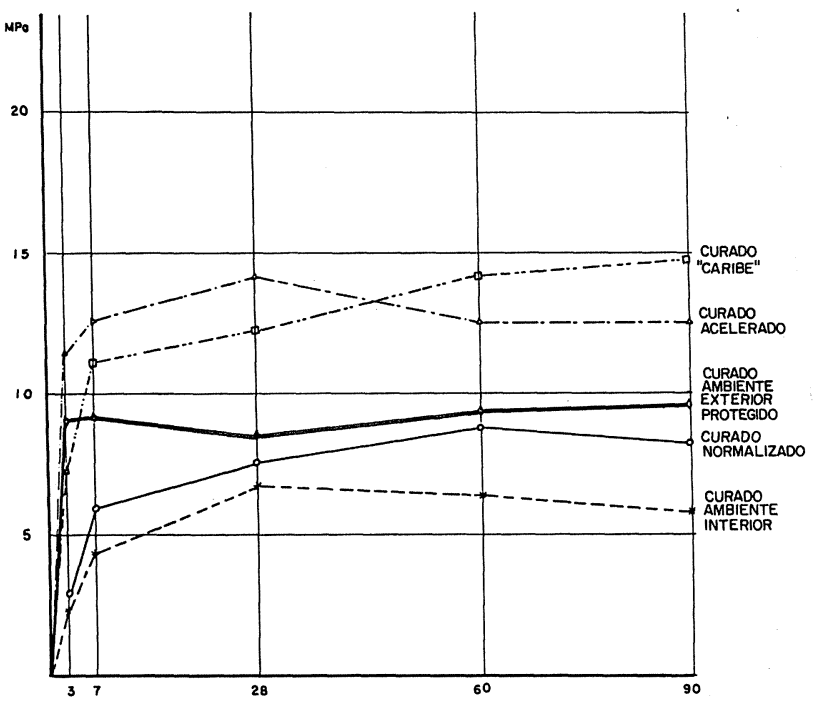

Fig. 14. - Influencia del tipo de curado en hormigones con un $50 \%$ de R.H.A. $(a /[c+c]=0,71)$.

\subsection{Hormigones con un $\mathbf{5 0} \%$ de RHA}

Manteniendo la misma relación agua/(cemento + ceniza) $=0,71$, se consiguieron las resistencias recogidas en la Fig. 14, para probetas con un $50 \%$ de RHA y un $50 \%$ de P-ARI-450.

Resulta palpable el efecto negativo, en cuanto a resistencia a compresión, al pasar del $33 \%$ al $50 \%$ del RHA, ya que en este caso, no se consigue superar los $15 \mathrm{MPa}$, en ningún caso ni para ninguna edad.

Al igual que en los casos de hormigones patrón y con $33 \%$ de RHA, sólo mediante el curado "acelerado" se consigue superar a tres dias los $10 \mathrm{MPa}$, lo que supone un dato importante de cara a posibles procesos de prefabricación, incluso para dosificaciones con un $50 \%$ de RHA.

Se confirma una ligera caida de resistencia, superados los 28 días, cuando se emplea el curado "acelerado", lo cual es acorde con la literatura existente para hormigones, a base de cemento Portland, mientras que sigue aumentando la resistencia en el curado "caribe" debido, posiblemente, a que en el "acelerado", transcurrido el segundo dia, la humedad relativa es de, aproximadamente, el $65 \%$, mientras que en el segundo caso prevalece constante el $80 \%$ de H.R.

Los resultados obtenidos con los otros tres tipos de curado, son francamente pobres, encontrándose prácticamente comprendidos en la zona $5 \mathrm{MPa} \div 10 \mathrm{MPa}$ para edades superiores a siete dias.

\subsection{Conclusiones parciales}

El proceso de curado se confirma como uno de los factores decisivos en la consecución de resistencias a compresión, especialmente importante al emplear dosificaciones con un $33 \%$ o $50 \%$ de RHA.

El curado "caribe" se muestra el más idóneo: por las altas resistencias conseguidas, por su crecimiento prácticamente continuado y su coste energético nulo, en los casos climatológicos con condiciones similares a las fijadas.

Los resultados conseguidos con la dosificación a base de un $33 \%$ de RHA (Fig. 13) ponen de manifiesto la importancia del proceso de curado, ampliando la zona de resistencias a compresión, que se sitúan entre $5 \mathrm{MPa}$ y 20 $\mathrm{MPa}$. Es decir, mediante los diferentes tipos de curado, se consiguen incrementos relativos que, en el caso de 90 días, llegan a ser del orden del $325 \%$ para idéntica dosificación.

\section{ANALISIS DE LA INFLUENCIA DEL CONTENIDO EN AGUA}

\subsection{Introducción}

Al mantener constante en 0,71 la relación de agua respecto al conglomerante (cemento 0 cemento y RHA), se constató, como era previsible, que disminuia la trabajabilidad al pasar del hormigón patrón al de $33 \%$ de RHA, y de éste al de $50 \%$ de RHA. Estaba programado -en base únicamente al curado "normalizado"comprobar la influencia del contenido de agua 


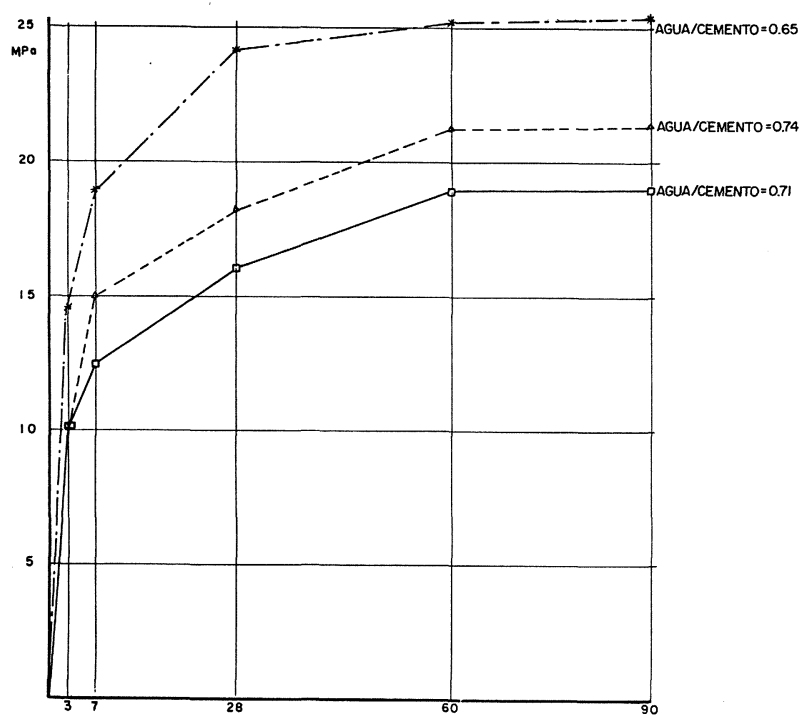

Fig. 15. - Análisis de la influencia del contenido en agua: hormigones patrón y curado normalizado.

en las tres dosificaciones, a base de mediciones de los descensos de cono y de la plasticidad. Los valores de los descensos son las medias de las resultantes en las dos amasadas necesarias para la dosificación de quince probetas.

\subsection{Hormigones patrones (Fig. 15)}

El hormigón de dosificación "patrón» (relación $a / c=0,71$ ) arrojó un valor del descenso de cono de $20 \mathrm{~mm}$. Mediante aumentos no muy importantes de agua, $(a / c=0,74)$ se midieron descensos considerables de cono, una media de $64 \mathrm{~mm}$, que arrojaron resistencias -a partir de tres dias- del orden de un $20 \%$, superiores a las de la "patrón".

Disminuyendo sensiblemente el agua $(a / c=$ $0,65)$ se presentaba una trabajabilidad deficiente, con descensos de cono del orden de $5 \mathrm{~mm}$, pero que arrojaron incrementos muy notables en las resistencias a compresión, respecto de la dosificación "patrón»: incrementos del orden del $35 \%$ a 28 dias y del $45 \%$ a 60 dias.

\subsection{Hormigones con un $33 \%$ de RHA (Fig. 16)}

La sensibilidad de estas dosificaciones al contenido de agua, resulta extraordinaria, lo cual era esperado, pues conociamos por trabajos anteriores (2) la avidez de agua de las cenizas RHA.

Para una relación $a /(C+c)=0,71$, el descenso de cono medio, resultó ser de $10 \mathrm{~mm}$, en lugar de los $20 \mathrm{~mm}$ de la «patrón». Se aumentó esta relación hasta 0,77 consiguiéndose descensos de unos $15 \mathrm{~mm}$ y experimentándose unos incrementos extraordinarios de resistencias a todas las edades, del orden del $90 \%$ a 28 dias, respecto de la dosificación de relación 0,71 , como puede comprobarse en la Fig. 16, debido a la mejor compactación del hormigón. Se incrementó el agua hasta un valor $\mathrm{a} /(\mathrm{C}+\mathrm{c})=$ 0,83 , lo que proporcionó una buena trabajabilidad, $40 \mathrm{~mm}$ de descenso, pero a costa de descensos notabilisimos de resistencias incluso, por debajo de los obtenidos con relación inferior, es decir, 0,71.

\subsection{Hormigones con un $50 \%$ de RHA} (Fig. 17)

Para la relación agua/conglomerante de partida: $a /(C+c)=0,71$, la trabajabilidad era pésima, arrojando descensos de cono prácticamente inapreciables.

Siguiendo la misma sistemática anterior, se adicionó una apreciable cantidad de agua, subiendo a relaciones de 0,79 y consiguiendo descensos de cono del orden de $15 \mathrm{~mm}$. Se siguió añadiendo agua hasta una relación de 0,86 (44 $\mathrm{mm}$ de descenso) y la evolución de sus resistencias a compresión se puede seguir en la Fig. 17, en la que se reflejan moderados incrementos en la resistencia para ambos incrementos de agua.

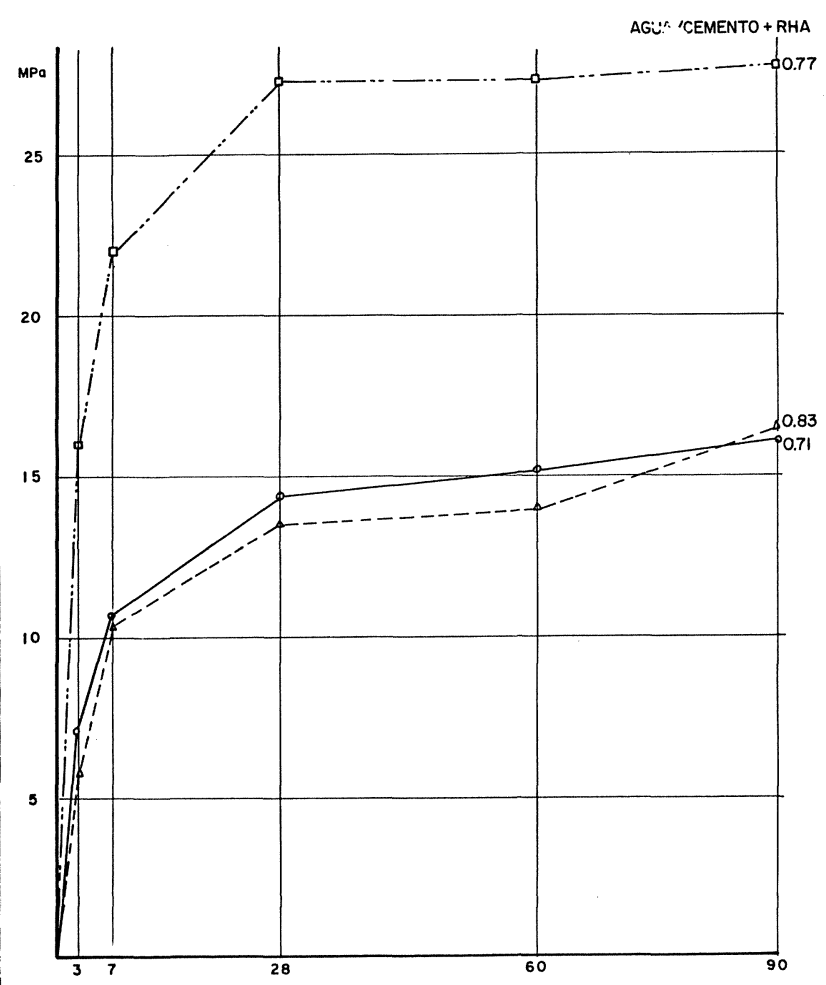

Fig. 16. - Analisis de la influencia del contenido en agua: hormigones con un $33 \%$ de R.H.A. y curado normalizado. 


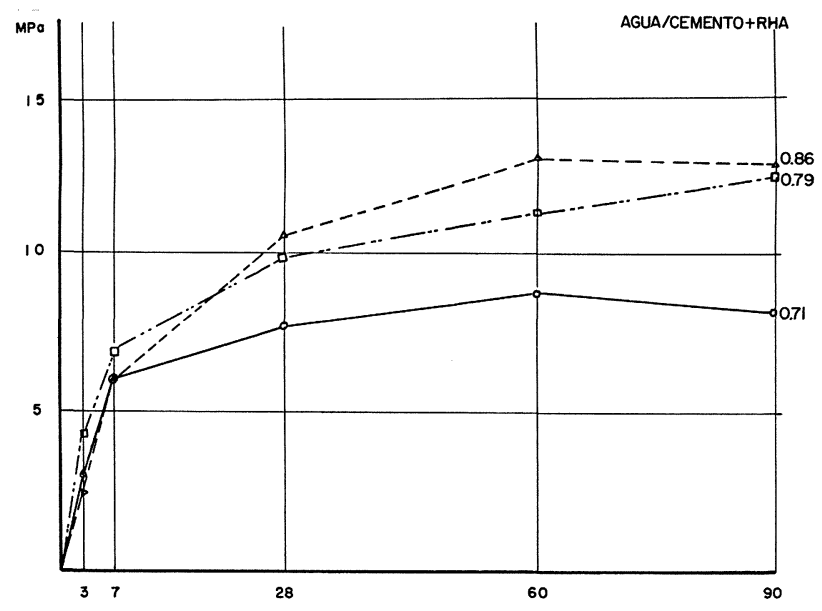

Fig. 17. - Análisis de la influencia del contenido en agua: hormigones con un $50 \%$ de R.H.A. y curado normalizado.

Para dosificaciones con un $50 \%$ de RHA y relaciones agua/conglomerante altas $(0,79$ y 0,86 ) se consiguen resistencias a 28 dias, del orden de 10 PMa con buena trabajabilidad.

\subsection{Conclusiones parciales}

Queda manifiesta la avidez de agua de los hormigones, a base de RHA respecto a los hormigones "patrón" a base de P-ARI-450 para equivalente trabajabilidad.

Resulta obvio, a la vista de los resultados recogidos en las figuras 15,16 y 17 , recomendar el estudio cuidadoso de la relación agua/conglomerante, ya que las variaciones de resistencia a compresión resultan notables.

Merece destacarse el extraordinario comportamiento de la dosificación a base de $33 \%$ de $\mathrm{RHA}$ y a/ $(\mathrm{C}+\mathrm{c})=0,77$, que proporciona resistencias superiores a $20 \mathrm{MPa}$ a 7 dias y superiores a $25 \mathrm{MPa}$, para edades superiores a los 28 dias, con descenso medio de cono de 15 $\mathrm{mm}$. Es decir, se consiguen con esta dosificación resistencias superiores a las de cualquiera de las dosificaciones "patrón" estudiadas.

\section{BIBLIOGRAFIA}

(1) ZORISLAV FRANJETIC: “Endurecimiento rápido del hormigón». Versión española de J. Calleja. Instituto E. Torroja. Madrid, 1971. 385 páginas.

(2) J. SALAS, M.a ISABEL SANCHEZ, PERCY CASTILLO Y JANER VERAS: «Empleo de cenizas de cáscara de arroz como adiciones en mortero". "Use of rice husk ash an admixture in mortar". Materiales de Construcción, n.o 203, págs. 23-41. Madrid, octubre 1986,

(3) JULIAN SALAS, MARINA ALVAREZ and JANER VERAS: "Lightweight insulating concretes with rice husk». The International Journal of Cement Composites. Vol. 8, Number 3. August, 1986. Págs. 171-180.

(4) K. W. NASSER and J. C. KENYON: "Why Not $3 \times$ 6 Inch Cylinders for Testing Concrete». ACl Journal, n.o 41 Proceedings V. 81. Págs. 47-53. January-February, 1984.

\section{publicación del i.e.t.c.c.}

\section{ACUEDUCTOS ROMANOS EN ESPAÑA \\ Carlos Fernández Casado}

Prof. Dr. Ing. de Caminos, Canales y Puertos

Esta publicación se compone de una serie de artículos, publicados en la Revista "Informes de la Construcción", en los cuales se hace un análisis de los acueductos romanos que existen en España y el balance de las condiciones de conservación en que se encuentra cada uno de ellos, incluyendo referencias históricas y literarias. Se ha llustrado con la reproducción de la valiosa documentación gráfica que posee el prestigioso autor.

Un volumen encuadernado en couché, a dos colores, de $21 \times 27$ centimetros, compuesto de 238 páginas, numerosos grabados, dibujos, fotos en blanco y negro y figuras de linea.

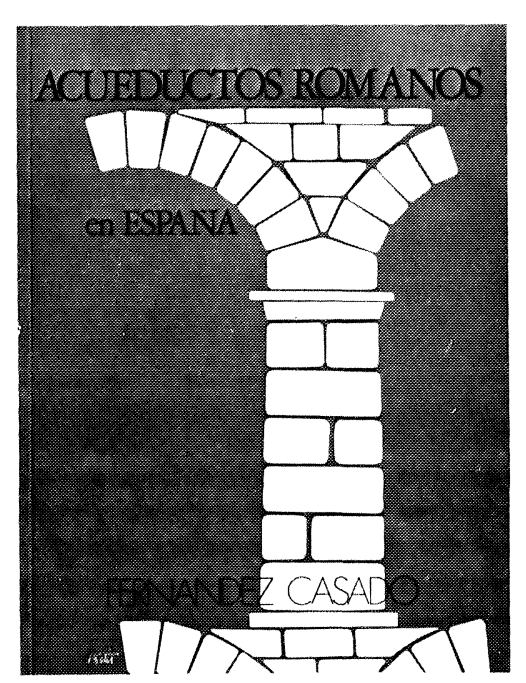

Precio: España, 1.500 ptas., 21 \$ UंSA. 